\title{
ECG as a Tool to Estimate Potassium and Calcium Concentrations in the Extracellular Space
}

\author{
Nicolas Pilia, Olaf Dössel, Gustavo Lenis*, Axel Loewe* \\ * shared senior authorship \\ Institute of Biomedical Engineering, Karlsruhe Institute of Technology, Karlsruhe, Germany
}

\begin{abstract}
Chronic kidney disease appears worldwide. In the United States, the number of patients suffering from kidney failure doubled from 1998 to 2010. A common treatment for these patients is haemodialysis. However, the frequency of deaths caused by cardiovascular diseases is up to $10 \%$ to $30 \%$ higher in patients undergoing dialysis than in the general population. To analyse the underlying effects and for a possible risk prediction, a continuous monitoring of the ionic concentrations that are influenced by dialysis is desired. In this work, a method for the reconstruction of the ionic concentrations of calcium and potassium from the ECG is proposed. In a first step, 91 monodomain simulations with the ten Tusscher ventricular cell model were performed for different extracellular ionic concentrations. From there, a standard 12-lead ECG was extracted. Calcium and potassium changes yielded ECGs clearly differing in amplitude and morphology. In a second step, the simulated ECG signals were used for reconstructing the ionic concentrations directly from the ECG. Features were extracted from the signals designed to describe changes caused by varied ionic concentrations. The inverse problem, i.e. coming back from the ECG features to the ionic concentrations was solved by regression with an artificial neural network. Results for potassium estimation yield an error of $0.00 \pm 0.28 \mathrm{mmol} / \mathrm{l}$ (mean \pm standard deviation) calculated with 7-fold cross validation. The estimation error for calcium was $0.00 \pm 0.08 \mathrm{mmol} / \mathrm{l}$. Although these results underline the suitability of the method, the used ECGs differed from the observed in a clinical environment. However, simulations allow an evaluation under controlled conditions of a particular effect that was intended to be investigated. As the application to clinical data is yet missing, this study can be seen as a proof of concept showing that an artificial neural network is capable of exactly estimating potassium and calcium concentrations from ECG features.
\end{abstract}

\section{Introduction}

Haemodialysis therapy is a common treatment method for patients suffering from chronic kidney disease (CKD) in the terminal stage. The amount of people in the United States suffering from kidney failure increased from 320,000 in 1998 to 650,000 in 2010 . The frequency of deaths caused by cardiovascular events within the dialysis patient group is up to $10 \%$ to $30 \%$ higher than in general population [1]. Patients suffering from end-stage CKD experience high variations of blood electrolyte concentrations. These can directly influence the functioning of the heart. Thus, research on cardiovascular links could improve therapy and risk stratification. One tool which is capable of capturing the electrophysiological properties of the heart in a non-invasive way is the electrocardiogram (ECG). It is known, that electrolyte concentrations of potassium $\left(\mathrm{K}^{+}\right)$and calcium $\left(\mathrm{Ca}^{2+}\right)$ affect the ECG [2]. Until now, a determination of the concentrations is connected to a blood test. Hence, continuous monitoring of the ionic concentration is impracticable. However, the ECG as a continuous, non-invasive monitoring tool could shed a light on the relation between heart diseases and changes in the ionic concentration particularly after leaving the strictly supervised clinical area where dialysis takes place, i.e allowing a monitoring at home. Articles have been published showing that the reconstruction of extracellular $\mathrm{K}^{+}$ concentration can be done using just one feature from the ECG with a quadratic regression [3]. In this study, we tried to estimate both $\mathrm{K}^{+}$and $\mathrm{Ca}^{2+}$ concentrations from the ECG. Therefore, we examined simulated ECGs at different concentration levels and designed features describing the observed changes in the ECG. A subset of these was used in connection with a machine learning method to reconstruct the concentrations.

\section{Methods}

\subsection{Simulations}

A total number of 91 computer simulations of the cardiac electrophysiology were performed at whole heart 
level. In the ten Tusscher ventricular cell model [4], the extracellular $\mathrm{K}^{+}$concentration $\left(\left[\mathrm{K}^{+}\right]_{\mathrm{o}}\right)$ is set to $5.4 \mathrm{mmol} / \mathrm{l}$ and the extracellular $\mathrm{Ca}^{2+}$ concentration $\left(\left[\mathrm{Ca}^{2+}\right]_{\mathrm{o}}\right)$ to $2.0 \mathrm{mmol} / \mathrm{l}$ by default. In this study, $\left[\mathrm{K}^{+}\right]_{\mathrm{o}}$ was varied between $3 \mathrm{mmol} / \mathrm{l}$ and $9 \mathrm{mmol} / \mathrm{l},\left[\mathrm{Ca}^{2+}\right]_{\mathrm{o}}$ between $0.6 \mathrm{mmol} / \mathrm{l}$ and $3 \mathrm{mmol} / 1$ in equally distributed steps. These ranges were also used in a previous simulation study evaluating changes in the atria and cover the clinically observed ranges [5]. Monodomain simulations and subsequent forward calculations using a torso model including various organs with different conductivities were performed with our simulation environment using an established model [6]. The standard 12-lead ECG was extracted with a samplerate of $1000 \mathrm{~Hz}$. Each computed ECG comprised a QRS complex and a $\mathrm{T}$ wave, but no $\mathrm{P}$ wave, since this was only a ventricular simulation. To calculate a feature value from the 12-lead ECG, the lead reduction method proposed in [7] was used. The transformation matrix was calculated by maximising the amplitude of a given part of the ECG, e.g. the T wave.

\subsection{Reconstruction of ionic concentrations}

As an input for the regression method, features were extracted from the ECG. Simulated ECGs for different $\left[\mathrm{Ca}^{2+}\right]_{\mathrm{o}}$ and $\left[\mathrm{K}^{+}\right]_{\mathrm{o}}$ are plotted in figures 1 and 2 . Changes caused by different ionic concentrations in the simulated ECGs were evaluated visually and features capturing these changes were designed (see figure 3 ). The canonical correlation analysis (CCA) was used to reduce features. The following features were used for the regression task: RT distance, $\mathrm{T}$ ascending slope, $\mathrm{T}$ descending slope, $\mathrm{T}$ ratio 1st half (ratio of energy of the first half of the $\mathrm{T}$ wave to the energy of the whole wave), $\mathrm{R}$ ratio amplitude to energy. The features $\mathrm{T}$ amplitude and $\mathrm{T}$ peakedness (2nd order coefficient of the quadratic fit for the peak of the $\mathrm{T}$ wave) were detected to be redundant by CCA. The 12-lead ECG signals were transformed twice: Features regarding the QRS complex were calculated from the transformed signal that maximised $\mathrm{R}$ peak amplitude, features regarding the $\mathrm{T}$ wave from the transformed signal maximising $\mathrm{T}$ wave amplitude.

A Bayesian neural network with early stopping [8] was chosen for reconstructing the ionic concentrations from the simulated ECGs. The implementation in MATLAB's Machine Learning Toolbox was used (MATLAB 2016b, The MathWorks, Inc., Natick, Massachusetts, United States). During training, randomly chosen setups were used for evaluating the early stopping criterion. If the error increased for 6 iterations, the training was stopped. The number of neurons and layers were chosen empirically. We allowed a maximum of 2 layers with up to 15 neurons in each layer. The estimation error was determined for each data partition of a 7-fold cross validation to eval- uate every possible network structure. Minimising the estimation error for each cross validation partition yielded 7 different optimal network structures for the available data. The structure that was chosen most often was finally selected. If there were two or more structures equally often chosen, the one with the least neurons was selected. With the optimal setting of layers and neurons per layer, the resulting network was evaluated again with 7-fold cross validation using the same data partitions as before. The setups used for the early stopping criterion were taken from the 6 cross validation training data groups left. Hence, the neural network was trained with the remaining 5 cross validation groups. Each group from the training data was used once as early stopping validation data and omitted for actual training. This yielded 91 x 6 (546) error values for each ion type. The training and the described evaluation was conducted 100 times, since artificial neural networks tend to run in a non-global local minimum during weight optimisation.

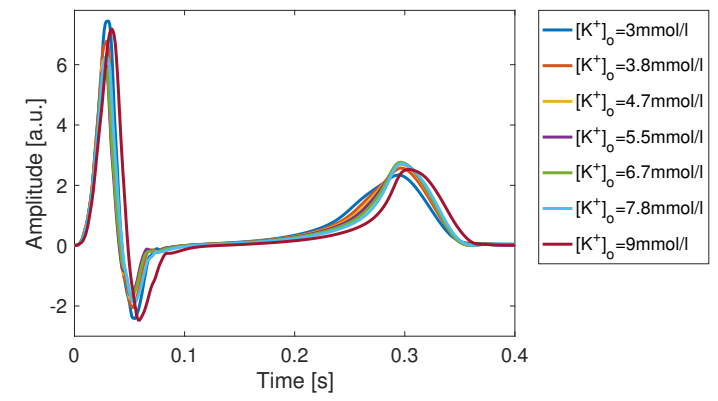

Figure 1. Simulated ECGs of different $\left[\mathrm{K}^{+}\right]_{\mathrm{o}}$ after lead reduction $\left(\left[\mathrm{Ca}^{2+}\right]_{\mathrm{o}}=1.8 \mathrm{mmol} / \mathrm{l}\right)$.

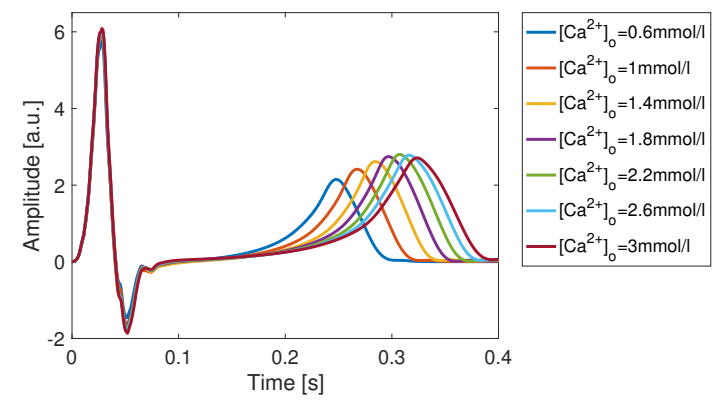

Figure 2. Simulated ECGs of different $\left[\mathrm{Ca}^{2+}\right]_{\mathrm{o}}$ after lead reduction $\left(\left[\mathrm{K}^{+}\right]_{\mathrm{o}}=5.5 \mathrm{mmol} / \mathrm{l}\right)$.

\section{Results}

The optimal neural network structure was found to be one layer and six neurons. The $\left[\mathrm{K}^{+}\right]_{\mathrm{o}}$ and $\left[\mathrm{Ca}^{2+}\right]_{\mathrm{o}}$ estimation errors for cross validation data, early stopping data and 


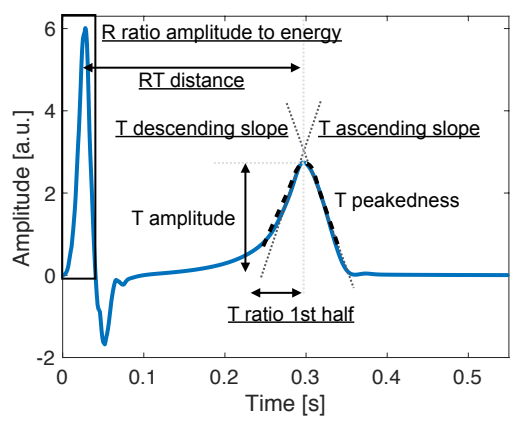

Figure 3. Features obtained from the ECG signals. The remaining features after reduction are underlined.

training data are displayed in the boxplot in figure 4 . The statistics of the cross validation errors of all 120 iterations yielded $0.00 \pm 0.28 \mathrm{mmol} / \mathrm{l}$ (mean \pm standard deviation) for $\left[\mathrm{K}^{+}\right]_{\mathrm{o}}$ estimation and $0.00 \pm 0.08 \mathrm{mmol} / \mathrm{l}$ for $\left[\mathrm{Ca}^{2+}\right]_{\mathrm{o}}$ estimation.

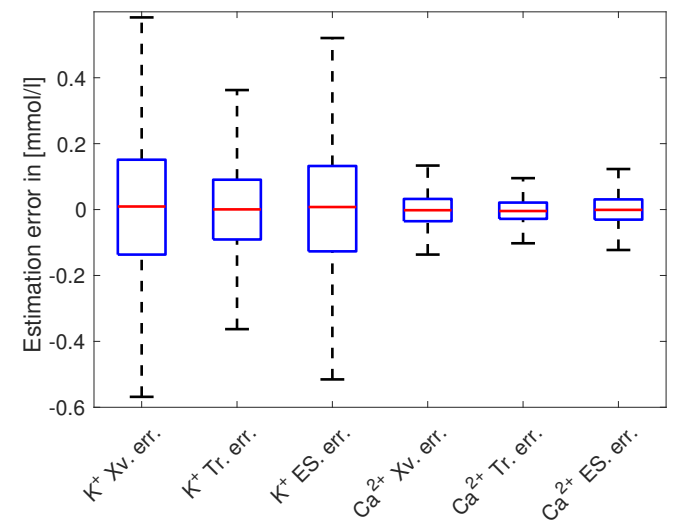

Figure 4. $\left[\mathrm{K}^{+}\right]_{\mathrm{o}}$ and $\left[\mathrm{Ca}^{2+}\right]_{\mathrm{o}}$ estimation errors of different data sets: Cross validation data (Xv.), Training data (Tr.) and early stopping criterion data (ES.). The red horizontal line is the median, the blue box determines the interquartile range. Outliers are not plotted.

\section{Discussion}

\subsection{Simulation method}

In this work, the ten Tusscher ventricular cell model was selected as one of a few human ventricular cell models with an advanced $\mathrm{Ca}^{2+}$ handling. Nevertheless, the O'Hara Rudy model offers a similarly sophisticated implementation, but it does not yield action potentials for $\left[\mathrm{K}^{+}\right]_{\mathrm{o}}$ as low as used here [9]. Himeno et al. [10] proposed a human ventricular cell model that offers a more realistic behaviour regarding a changed $\left[\mathrm{Ca}^{2+}\right]_{\mathrm{o}}$.
Although changes in the ECGs dependent on $\left[\mathrm{Ca}^{2+}\right]_{\mathrm{o}}$ and $\left[\mathrm{K}^{+}\right]_{0}$ were clearly visible, limitations regarding the accuracy of the ECGs were discovered during the study: A prolongation/shortening of the QT interval, that is often seen in a clinical environment and that was described in [2], could not be reproduced for severe hypocalcaemia/hypercalcaemia, respectively. The simulations showed exactly the reverse effect. An imbalance of $\left[\mathrm{K}^{+}\right]_{\mathrm{o}}$ resulted in a change of the ECG that is partly in accordance to literature. In [11], changes due to hyperkalaemia were identified: an increase of the peakedness and amplitude of the $\mathrm{T}$ wave and a widening of the QRS complex. All phenomena were visible in the simulated ECGs but changes were partly not as prominent as visualised in [11]. In [2], a decrease of the QT interval is also named as a marker for hyperkalaemia. In this study, an increase of $\left[\mathrm{K}^{+}\right]_{\mathrm{o}}$ showed a reverse effect on the comparable marker RT interval. However, it is well-known that a change of the QT interval can have different reasons. In addition, in [2], it is stated that hypokalaemia induces ST depression, $\mathrm{T}$ wave inversion and $\mathrm{T}$ wave amplitude decrease which could not be reproduced here.

Some further effects could not be evaluated, since the simulation method was not able to account for them. In this particular simulation study, the Purkinje fibres were not directly modelled. Rather, a phenomenological modelling neglecting the influence of changed ionic concentrations [12] was used. Moreover, changes in atrial electrophysiology could not be evaluated because a ventricular cell model was used. However, P wave changes are reported in [13]. Changes in the conduction properties of the AV node described in [2] were also not reproducible. In addition, arrhythmia triggering phenomena caused by a changed $\left[\mathrm{K}^{+}\right]_{\mathrm{o}}$ (as reported in [2]) could not be observed. Possible inter-subject changes on the anatomical and physiological level could not be reproduced in the simulations since just one anatomical and physiological model was used. As some changes visible in clinical routine are patient dependent, the main advantage of a simulation study is underlined: it offers the opportunity to eliminate all other factors and focus on the pure influence of the ones to be evaluated. So, the pure effects caused by the changed ionic concentrations were analysed and described.

\subsection{Regression method}

An artificial neural network was shown to be an appropriate regression method for the reconstruction of the ionic concentrations from the ECG. The standard deviation of the $\left[\mathrm{K}^{+}\right]_{\mathrm{o}}$ estimation error was $0.28 \mathrm{mmol} / \mathrm{l}$. This is a small value compared to both the $\left[\mathrm{K}^{+}\right]_{\text {o }}$ concentration steps in the simulations which were about $0.42 \mathrm{mmol} / \mathrm{l}$ and the total range of $\left[\mathrm{K}^{+}\right]_{\mathrm{o}}$ of $6 \mathrm{mmol} / \mathrm{l}$. The standard deviation of the $\left[\mathrm{Ca}^{2+}\right]_{\text {o }}$ estimation error was $0.08 \mathrm{mmol} / \mathrm{l}$ which has to be 
seen alongside the $\left[\mathrm{Ca}^{2+}\right]_{\mathrm{o}}$ steps of $0.20 \mathrm{mmol} / 1$ and the total variation of $2.4 \mathrm{mmol} / \mathrm{l}$.

The problem of overfitting can always appear in the field of machine learning. Although regularisation methods were applied and the network structure was relatively simple, we cannot exclude overfitting for sure. The problem arises in the form of a bad performance on unseen data compared to the training data. So we tried to quantify this by also evaluating the training data with the proposed neural network. Comparing the cross validation error and the training error visualised in figure 4 , we see that median values are both around zero. As expected, interquartile ranges are smaller with the training data than with cross validation or early stopping data. However, the interquartile range of the cross validation data was not dramatically larger. We therefore assume, that overfitting was at least small.

\section{Conclusion and Outlook}

In this work, a simulation study was performed to systematically study the influence of changed ionic concentrations on the ECG. The results partly confirmed findings in literature. We could show that artificial neural networks are capable of reconstructing the ionic concentrations with the extracted features from the simulated ECGs as inputs.

A few topics of this work need further research: Starting from the selection of the cell model, others could be evaluated. A model optimisation of the O'Hara Rudy model could correct the undesired behaviour for low $\left[\mathrm{K}^{+}\right]_{0}$. Himeno's ventricular cell model [10] could be used as electrophysical cell model, too. Furthermore, due to different heart and torso geometries, a change of these could result in other feature value trends. Thus, an expansion using more anatomical models is crucial for further evaluation of the findings. In [3], a patient specific calibration is introduced to improve results on patient data. This substantiates a patient-dependency of at least their evaluated feature, but could also mean that a calibration is needed in general. Another important point would be the extension of the study featuring an atrial model. This could deliver more features obtained from the $\mathrm{P}$ wave. These might improve the estimation of $\left[\mathrm{K}^{+}\right]_{\mathrm{o}}$. The determination of the features was done by looking at changes in the temporal ECG signals. This could be extended to an evaluation of the frequency domain or the principal components, for example. Furthermore, the application of the method to clinical data is still missing. Many diseases can cause shifts in ECG parameters. Thus, a variety of patients showing abnormal $\left[\mathrm{K}^{+}\right]_{\mathrm{o}}$ and $\left[\mathrm{Ca}^{2+}\right]_{0}$ should be included during the data acquisition. This could deliver more information about the dependency of ionic concentrations and ECG markers, that are assumed to exist. However, this study was a first step on the way to a reliably working method for the estimation of ionic concentrations. By achieving small estimation er- rors, we proofed that our features describe the changes in ECG and that a simple neural network, which was especially designed to prevent overfitting, with one layer and six neurons can reconstruct $\left[\mathrm{K}^{+}\right]_{\mathrm{o}}$ and $\left[\mathrm{Ca}^{2+}\right]_{\mathrm{o}}$.

\section{References}

[1] Sarnak MJ, Levey AS, Schoolwerth AC, et. al. Kidney disease as a risk factor for development of cardiovascular disease. Circulation 2003;108(17):2154-2169.

[2] Van Mieghem C, Sabbe M, Knockaert D. The clinical value of the ECG in noncardiac conditions. Chest 2004; 125(4):1561-1576.

[3] Corsi C, Cortesi M, Callisesi G, et. al. Noninvasive quantification of blood potassium concentration from ECG in hemodialysis patients. Scientific Reports 2017;7:42492.

[4] ten Tusscher KHWJ, Panfilov AV. Alternans and spiral breakup in a human ventricular tissue model. American Journal of Physiology Heart and Circulatory Physiology 2006;291(3):H1088-H1100.

[5] Passini E, Genovesi S, Severi S. Human atrial cell models to analyse haemodialysis-related effects on cardiac electrophysiology: Work in progress. Computational and Mathematical Methods in Medicine 2014;2014:1-18.

[6] Keller DUJ, Weiss DL, Dössel O, Seemann G. Influence of $\mathrm{I}(\mathrm{Ks})$ heterogeneities on the genesis of the T-wave: a computational evaluation. IEEE Transactions on Biomedical Engineering 2012;59(2):311-322.

[7] Lenis G, Lutz Y, Seeman G, et. al. Post extrasystolic T wave change in subjects with structural healthy ventricles? Measurement and simulation. In Computing in Cardiology, volume 41. IEEE, 2014; 1069-1072.

[8] Bishop C. Pattern recognition and machine learning. Information science and statistics (Computer science). New York, NY: Springer, 2006.

[9] Kharche S, Callisesi G, Stary T, Bracci A, Severi S. Simulating the effects of serum potassium on the ECG. In Computing in Cardiology. IEEE, 2012; 225-228.

[10] Himeno Y, Asakura K, Cha CY, et. al. A human ventricular myocyte model with a refined representation of excitationcontraction coupling. Biophysical Journal 2015;109(2):415 $-427$.

[11] Webster A, Brady W, Morris F. Recognising signs of danger: ECG changes resulting from an abnormal serum potassium concentration. Emergency Medicine Journal Jan 2002;19(1):74-77.

[12] Poole-Wilson PA. Potassium and the heart. Clinics in endocrinology and metabolism 1984;13(2):249-268.

[13] Krueger MW, Severi S, Rhode K, et. al. Alterations of atrial electrophysiology related to hemodialysis session: insights from a multiscale computer model. Journal of Electrocardiology 2011;44(2):176-183.

Address for correspondence:

Nicolas Pilia

Institute for Biomedical Engineering (IBT), KIT

Fritz-Haber-Weg 1, 76131 Karlsruhe, Germany

publications@ibt.kit.edu 